\title{
The BRAF V600E mutation is a predictor of the effect of radioiodine therapy in papillary thyroid cancer
}

Junshang Ge1,2,3, Jie Wang2², Hui Wang1, Xianjie Jiang'2, Qianjin Liao'1, Qian Gong1, Yongzhen $\mathrm{Mo}^{2}$, Xiaoling $\mathrm{Li}^{1,2,3}$, Guiyuan Li1 ${ }^{1,2,3}$, Wei Xiong ${ }^{1,2,3}$, Jin Zhao ${ }^{1,2}$, Zhaoyang Zeng ${ }^{1,2,3}$

1. NHC Key Laboratory of Carcinogenesis (Central South University) and Hunan Key Laboratory of Translational Radiation Oncology, Hunan Cancer Hospital and the Affiliated Cancer Hospital of Xiangya School of Medicine, Central South University, Changsha, Hunan, China;

2. Key Laboratory of Carcinogenesis and Cancer Invasion of the Chinese Ministry of Education, Cancer Research Institute and School of Basic Medicine, Central South University, Changsha, Hunan, China;

3. Hunan Key Laboratory of Nonresolving Inflammation and Cancer, Disease Genome Research Center, the Third Xiangya Hospital, Central South University, Changsha, Hunan, China.

\Corresponding authors: Prof. Zhaoyang Zeng, Email: zengzhaoyang@csu.edu.cn, and Dr. Jin Zhao, Email: 709833789@qq.com.

(c) The author(s). This is an open access article distributed under the terms of the Creative Commons Attribution License (https://creativecommons.org/licenses/by/4.0/). See http://ivyspring.com/terms for full terms and conditions.

Received: 2019.01.13; Accepted: 2019.10.20; Published: 2020.01.01

\begin{abstract}
Objective: To investigate the correlation between the BRAF V600E gene mutation and clinicopathological features and thyroid function after iodine-131 treatment in patients with papillary thyroid cancer (PTC).

Methods: A total of 128 PTC patients who underwent iodine-131 treatment after a total thyroidectomy from February 2015 to November 2016 at Hunan Cancer Hospital, China, were recruited. There were 25 males and 103 females. The age range was 11 to 73 years old. The BRAF V600E mutation in tumor tissues was detected by amplification-restriction mutation system polymerase chain reaction (ARMS-PCR), and the serum levels of $\mathrm{Tg}, \mathrm{TSH}, \mathrm{Tg}-\mathrm{Ab}$, and $\mathrm{Tpo}-\mathrm{Ab}$ were measured by chemiluminescence after iodine-131 treatment. The BRAF V600E mutation was shown to be associated with clinicopathological characteristics and thyroid function indicators after iodine-131 treatment.

Results: BRAF V600E mutation was detected in 75 of the 128 patients $(58.6 \%)$ and was observed more frequently in cases with elevated $T g$ levels $(T g>1.00)$ at 3, 6, 12, and 18 months after treatment compared with patients without any BRAF mutations $(P<0.05)$. Patients with BRAF V600E mutation had significant lower level of $\mathrm{Tg}$-Ab at 3 and 12 months after treatment with iodine-131 than patients without BRAF V600E mutation $(P<0.05)$. Among the 75 BRAF V600E patients, no significant association was found between the levels of TSH and Tpo-Ab after iodine-131 treatment $(P>0.05)$. The BRAF V600E mutation was closely associated with the high-risk and age of the patient ( $\geq 45$ years old) $(P<0.05)$, but there was no significant correlation with gender, clinical stage, and distant metastasis.

Conclusion: The BRAF V600E mutation is closely related to serum $\mathrm{Tg}$ elevation after treatment with iodine-131 in papillary thyroid cancer. These findings suggest that this BRAF mutation may be a predictor of the efficacy of iodine-131 treatment for papillary thyroid cancer.
\end{abstract}

Key words: I -131 treatment, BRAF V600E, Thyroid function, papillary thyroid carcinoma

\section{Introduction}

Thyroid cancer is the most common malignant tumor of the endocrine system and the most common head and neck cancer. The incidence of thyroid cancer has increased significantly in the past two decades[1].
Thyroid cancer primarily includes the following four pathological types: papillary thyroid carcinoma, follicular thyroid carcinoma, undifferentiated carcinoma and medullary thyroid carcinoma. Among 
these types, papillary thyroid carcinoma is the most common, accounting for $85 \%$ to $90 \%$ of the total incidences. Due to the relatively inert biological behavior of PTC, the overall prognosis is relatively good, and the 10-year survival rate of patients is greater than $90 \%$. However, many cases still have a poor prognosis, any many patients eventually die from postoperative recurrence and metastasis. Therefore, predicting the prognosis of papillary thyroid carcinoma and customizing individualized treatment programs have become topics of concern for clinicians.

At present, surgical resection is the preferred treatment for papillary thyroid carcinoma, and this treatment is widely used in clinical practice[2]. After thyroidectomy, adjuvant treatment is usually given, depending on the severity of the condition and the metastasis of the tumor. Among the various adjuvant treatments, I-131 adjuvant radiotherapy is the most common. Thyroid tissue-derived tumor cells usually retain the ability to take up iodine. I-131 treatment can effectively eliminate residual thyroid tissue and micrometastases; therefore, this treatment plays an important role in reducing the recurrence rate and improving survival[3]. Predictive evaluation of the therapeutic effect of I-131 treatment and the development of a corresponding degree of chemotherapy can effectively improve the prognosis of patients with PTC and prolong the recurrence-free survival of patients.

The BRAF gene is located on human chromosome $7 \mathrm{q} 34$ and encodes a serine/threonine protein kinase with a molecular weight of $67 \mathrm{kD}-99$ $\mathrm{kDs}[4]$. This protein kinase is involved in regulating the transcriptional activity of cells during cell growth, division and differentiation, and it affects the regulation of cell morphology and the distribution of the cytoskeleton. Approximately $80 \%$ of the $B R A F$ mutations are an A-T point mutation at the bp 1799, which is known as the BRAF V600E mutation. BRAF gene mutations are associated with the development of a variety of tumors, such as melanoma[5], lung cancer[6], colorectal cancer[7] and thyroid cancer. In recent years, a large body of literature has shown that the BRAF V600E mutation is associated with a series of adverse clinical pathological factors in PTC, such as advanced disease, invasive characteristics, and others[8-14]. Many studies have shown that the BRAF V600E mutation may result in the activation of downstream genes of the MAPK pathway, which may lead to a slight downregulation of the sodium iodide symporter (NIS), thereby causing an inaccurate NIS localization wherein the NIS cannot be accurately localized to the cell membrane[15, 16]. This failure of proper localization leads to a decrease in the ability of tumor cells to take up iodine[17, 18]. I-131 adjuvant radiotherapy relies on the uptake of radioactive iodine by tumor cells; therefore, the BRAF V600E mutation may lead to a decrease in the therapeutic effect of iodine-131, resulting in a poor prognosis.

The clinical evaluation of the efficacy of I-131 treatment after total thyroid cancer surgery includes imaging examination and tests for thyroid function[19]. The evaluation of the efficacy of I-131 treatment in the thyroid index primarily includes thyroglobulin (Tg), thyroid stimulating hormone (TSH), free T3 (FT3), free T4 (FT4), thyroid peroxidase antibody (TPO-Ab) and thyroglobulin antibody (TG$\mathrm{Ab}$ ). Among these factors, dynamic monitoring of thyroglobulin (Tg) levels is important for postoperative follow-up and prognosis evaluation, and such monitoring has been widely used in clinical practice $[20,21]$. Thyroglobulin glycoprotein is derived from functional thyroid tissue. Under the condition of thyroidectomy, if other physical and chemical conditions are stable, the serum Tg level is directly proportional to the size of thyroid residual tissue.

We primarily investigated the relationship among the BRAF V600E gene mutation, the clinicopathological features of PTC patients, and the levels of the thyroid function indicators $\mathrm{Tg}, \mathrm{TSH}$, $\mathrm{Tg}-\mathrm{Ab}$ and Tpo-Ab after iodine-131 treatment.

\section{Materials and Methods}

\section{Materials}

A cohort of 128 patients with PTC who underwent I-131 treatment following total thyroidectomy at Hunan Cancer Hospital from February 2015 to November 2016 were recruited. Patients were eligible for enrolment into the cohort if they had primary papillary thyroid carcinoma and received total thyroidectomy and postoperative iodine-131 treatment. The main exclusion criteria were a diagnosis of double primary cancer and poor physical condition. Written informed consents were obtained from all patients.

There were 25 male and 103 female patients with an age range of 11 to 73 years and a median age of 40 years. According to the International Anti-Cancer Alliance/United States Cancer Society (UICC/AJCC) 2008 staging criteria, 68 cases were stage I, 8 cases were stage II, 8 cases were stage III, and 38 cases were stage IV; the stages of the remaining 6 cases were unknown.

\section{Methods}

Total thyroidectomy and iodine- 131 treatment were performed in all cases, and TSH inhibition was performed according to the ATA treatment guidelines. The BRAF V600E mutation was detected by 
amplification-blocking mutation system polymerase chain reaction (ARMS-PCR). The serum levels of $\mathrm{Tg}$, $\mathrm{TSH}, \mathrm{Tg}-\mathrm{Ab}$ and Tpo-Ab were detected by chemiluminescence after the administration of iodine-131 treatment. All testing procedures were performed in compliance with quality control standards.

\section{Data analysis}

The statistical software SPSS for Windows was used for the statistical analyses. The Pearson's chisquare test or a t-test were used to analyze the relationships among the presence of the $B R A F$ mutation, the clinicopathological features of patients and the results of the thyroid function tests. When $P<0.05$, differences were considered statistically significant.

\section{Results}

\section{Relationship between the BRAFV600E mutation and clinicopathological features}

Among all the patients, 75 cases were positive for the BRAF V600E mutation, and 53 patients carried the wild type $B R A F$ gene, thus the mutation rate was $58.6 \%$. Among the patients with the BRAF V600E mutation, there were 17 males and 58 females; among the patients with wild-type $B R A F$, there were 8 males and 47 females $(P=0.25)$. The median age of patients with the BRAF mutation was 41 years, while the median age of patients with wild-type $B R A F$ was 38 years $(P=0.12)$. The BRAF V600E mutation was closely associated with the high-risk age ( $\geq 45$ years) $(P=0.025)$. There was no significant correlation between the BRAF V600E mutation and lymph node metastasis $(P=0.059)$, distant metastasis $(P=0.76)$, or clinical stage $(P=0.26)$ (Table 1$)$.

\section{BRAFV600E mutation and thyroid function following iodine-131 treatment}

The $\mathrm{Tg}$ levels at 3, 6, 12, and 18 months after iodine-131 treatment in patients with the BRAF mutation were significantly higher compared with those in patients with wild-type BRAF $(P<0.05)$ (Table 2, Fig. 1). BRAF V600E-mutation-patients compared with BRAF V600E-WT-patients, under 3 months treatment, the mean Tg level was $3.02 \mu \mathrm{g} / \mathrm{L}$ vs 0.54 $\mu \mathrm{g} / \mathrm{L}$, and the median Tg level was $0.54 \mu \mathrm{g} / \mathrm{L}$ vs 0.04 $\mu \mathrm{g} / \mathrm{L}(P=0.004)$; at 6 months after treatment, the mean $\mathrm{Tg}$ level was $2.50 \mu \mathrm{g} / \mathrm{L}$ vs $0.51 \mu \mathrm{g} / \mathrm{L}$, and the median $\mathrm{Tg}$ level was $0.30 \mu \mathrm{g} / \mathrm{L}$ vs $0.04 \mu \mathrm{g} / \mathrm{L}(P=0.03)$; at 12 months after treatment, the mean $\mathrm{Tg}$ level was 3.65 $\mu \mathrm{g} / \mathrm{L}$ vs $0.39 \mu \mathrm{g} / \mathrm{L}$, and the median Tg level was 0.26 $\mu \mathrm{g} / \mathrm{L}$ vs $0.04 \mu \mathrm{g} / \mathrm{L}(\mathrm{P}=0.010) ;$ and at 18 months after treatment, the mean $\mathrm{Tg}$ level was $1.74 \mu \mathrm{g} / \mathrm{L}$ vs 0.18 $\mu \mathrm{g} / \mathrm{L}$, and the median Tg level was $0.28 \mu \mathrm{g} / \mathrm{L}$ vs 0.04 $\mu \mathrm{g} / \mathrm{L} \quad(P=0.015)$. The rate of elevated $\mathrm{Tg}$ levels
$(\mathrm{Tg}>1.00)$ at $3,6,12$, and 18 months after treatment was significantly higher in patients with the $B R A F$ mutation than in patients with wild-type BRAF $(44.5 \%$ vs $15.2 \%, 29.7 \%$ vs $10.4 \%, 27.8 \%$ vs $11.1 \%, 27.6 \%$ vs $5.1 \%)(P<0.05)$ (Table 2, Fig. 2). The level of $\mathrm{Tg}-\mathrm{Ab}$ at 3 and 12 months after treatment with iodine-131 in patients with the BRAF mutation was significantly lower than in patients with wild-type BRAF $(P<0.05)$ (Table 2, Fig. 3). The mean level of Tg-Ab was 94.10 $\mathrm{IU} / \mathrm{mL}$ vs $274.85 \mathrm{IU} / \mathrm{mL}$, and the median $\mathrm{Tg}$-Ab level was $16.31 \mathrm{IU} / \mathrm{mL}$ vs $26.93 \mathrm{IU} / \mathrm{mL}(P=0.048)$. At 12 months after treatment, the mean $\mathrm{Tg}-\mathrm{Ab}$ level was $52.74 \mathrm{IU} / \mathrm{mL}$ vs 241.31 . IU $/ \mathrm{mL}$, and the median $\mathrm{Tg}-\mathrm{Ab}$ level was $13.47 \mathrm{IU} / \mathrm{mL}$ vs $17.46 \mathrm{IU} / \mathrm{mL}$ $(P=0.036)$. Due to the tracking time limit, there were only 36 cases that could be traced to 24 months after treatment, including 23 cases of BRAF-positive patients and 13 cases of patients with wild-type BRAF. No significant differences were detected between $B R A F$ mutational status and thyroid function parameters, which may be due to the low number of cases $(\mathrm{Tg}$ mean $=1.78 \mu \mathrm{g} / \mathrm{L}$ vs $0.21 \mu \mathrm{g} / \mathrm{L}$, median $=0.40$ $\mu \mathrm{g} / \mathrm{L}$ vs $0.04 \mu \mathrm{g} / \mathrm{L}, P=0.079)$. There was no correlation between BRAF V600E mutational status and the levels of TSH and Tpo-Ab after iodine 131-treatment $(P>0.05)$ (Table 2).

\section{Clinicopathological features and thyroid function following iodine-131 treatment}

Based on our sample size and follow-up time, there was no correlation between thyroid function and patient's age, gender, clinical stage, lymphovascular invasion, and metastasis $(P>0.05)$.

Table 1. Comparison of BRAF V600E mutation status and clinicopathological features of 128 patients with PTC.

\begin{tabular}{|c|c|c|c|c|}
\hline \multirow[t]{2}{*}{ Variables } & \multirow{2}{*}{$\begin{array}{l}\text { No. of } \\
\text { patients }\end{array}$} & \multicolumn{2}{|c|}{$B R A F$ mutation } & \multirow[t]{2}{*}{$P$ value } \\
\hline & & Positive & Negative & \\
\hline \multicolumn{5}{|l|}{ Age, year } \\
\hline$<45$ & 82 & 42 & 40 & 0.025 \\
\hline$\geq 45$ & 46 & 33 & 13 & \\
\hline \multicolumn{5}{|l|}{ Gender } \\
\hline Male & 25 & 17 & 8 & 0.25 \\
\hline Female & 103 & 58 & 47 & \\
\hline \multicolumn{5}{|c|}{ Clinical stage } \\
\hline I & 68 & 38 & 30 & 0.26 \\
\hline II & 8 & 3 & 5 & \\
\hline III & 8 & 5 & 3 & \\
\hline IV & 38 & 26 & 12 & \\
\hline NA & 6 & 3 & 3 & \\
\hline I+ III & 76 & 41 & 35 & 0.14 \\
\hline III+IV & 46 & 31 & 15 & \\
\hline \multicolumn{5}{|c|}{ Lymphovascular Invasion } \\
\hline No & 7 & 2 & 5 & 0.059 \\
\hline Yes & 121 & 73 & 48 & \\
\hline \multicolumn{5}{|l|}{ Metastasis } \\
\hline No & 117 & 69 & 48 & 0.76 \\
\hline Yes & 11 & 6 & 5 & \\
\hline
\end{tabular}


Table 2. Correlation between BRAF mutation status and thyroid function in 128 patients with papillary thyroid carcinoma.

\begin{tabular}{|c|c|c|c|c|c|c|c|c|c|c|c|c|c|c|c|}
\hline \multirow[t]{2}{*}{ Test items } & \multicolumn{2}{|c|}{3 months review } & \multirow{2}{*}{$\begin{array}{l}P \\
\text { value }\end{array}$} & \multicolumn{2}{|c|}{6 months review } & \multirow{2}{*}{$\begin{array}{l}P \\
\text { value }\end{array}$} & \multicolumn{2}{|c|}{12 months review } & \multirow{2}{*}{$\begin{array}{l}P \\
\text { value }\end{array}$} & \multicolumn{2}{|c|}{18 months review } & \multirow{2}{*}{$\begin{array}{l}P \\
\text { value }\end{array}$} & \multicolumn{2}{|c|}{24 months review } & \multirow{2}{*}{$\begin{array}{l}P \\
\text { value }\end{array}$} \\
\hline & $B R A F+$ & $B R A F-$ & & $B R A F+$ & BRAF- & & $B R A F+$ & BRAF- & & BRAF+ & BRAF- & & BRAF+ & $B R A F-$ & \\
\hline Tg Average $(\mu \mathrm{g} / \mathrm{L})$ & 3.02 & 0.54 & 0.004 & 2.50 & 0.51 & 0.03 & 3.65 & 0.39 & 0.010 & 1.74 & 0.18 & 0.015 & 1.78 & 0.21 & 0.079 \\
\hline $\operatorname{Tg}$ Median $(\mu \mathrm{g} / \mathrm{L})$ & 0.54 & 0.04 & & 0.30 & 0.04 & & 0.26 & 0.04 & & 0.28 & 0.04 & & 0.40 & 0.04 & \\
\hline Rate of elevated $\mathrm{Tg}(\geq 1.00)$ & $42.7 \%$ & $9.4 \%$ & $<0.001$ & $29.7 \%$ & $10.4 \%$ & 0.025 & $28.3 \%$ & $10.0 \%$ & 0.016 & $27.6 \%$ & $5.1 \%$ & 0.036 & $30.4 \%$ & $7.7 \%$ & 0.25 \\
\hline TSH Average (IU/mL) & 0.930 & 0.780 & 0.45 & 0.370 & 0.290 & 0.68 & 1.098 & 1.555 & 0.65 & 0.222 & 0.281 & 0.60 & 0.398 & 0.107 & 0.60 \\
\hline TSH Median (IU/mL) & 0.114 & 0.082 & & 0.072 & 0.036 & & 0.066 & 0.082 & & 0.0545 & 0.0505 & & 0.101 & 0.042 & \\
\hline Tg-Ab Average (IU/mL) & 94.10 & 274.85 & 0.048 & 115.05 & 233.95 & 0.29 & 52.74 & 241.31 & 0.036 & 131.49 & 257.77 & 0.09 & 293.31 & 445.07 & 0.66 \\
\hline Tg-Ab Median (IU/mL) & 16.31 & 26.93 & & 14.68 & 22.05 & & 13.47 & 17.46 & & 14.08 & 18.99 & & 14.15 & 21.85 & \\
\hline
\end{tabular}

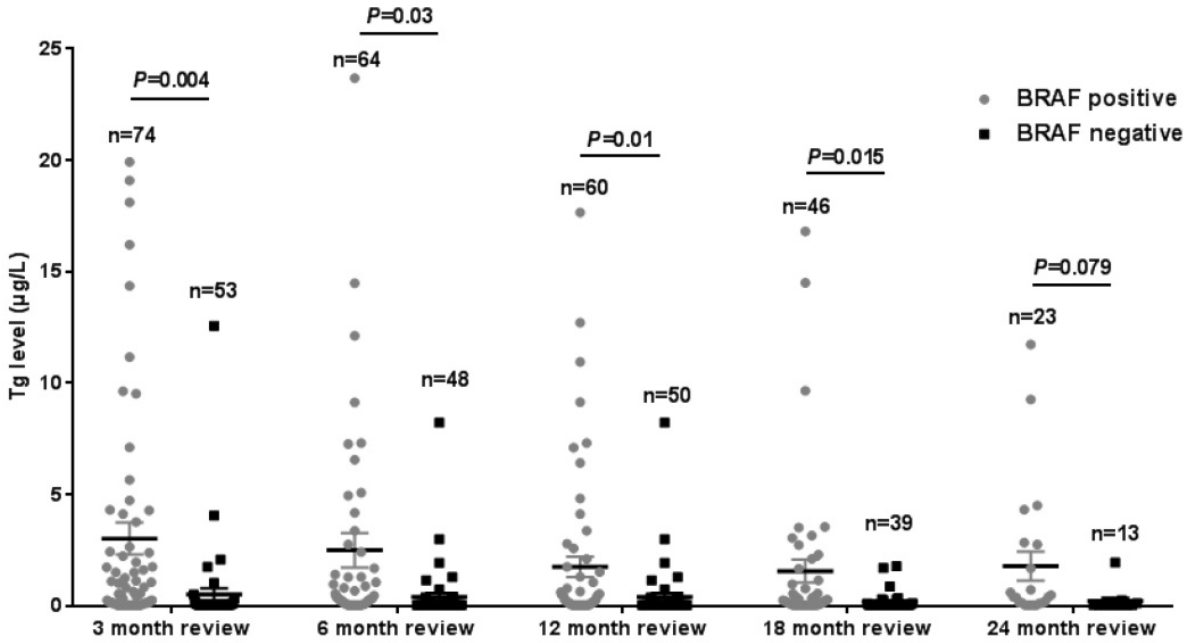

Result of Thyroid Function Tests

Figure 1. Tg levels in the 3rd, 6th, 12th, 18th, and 24th months after thyroid cancer treatment with iodine-131 in 128 patients from Hunan Cancer Hospital (error bar: mean with SEM).
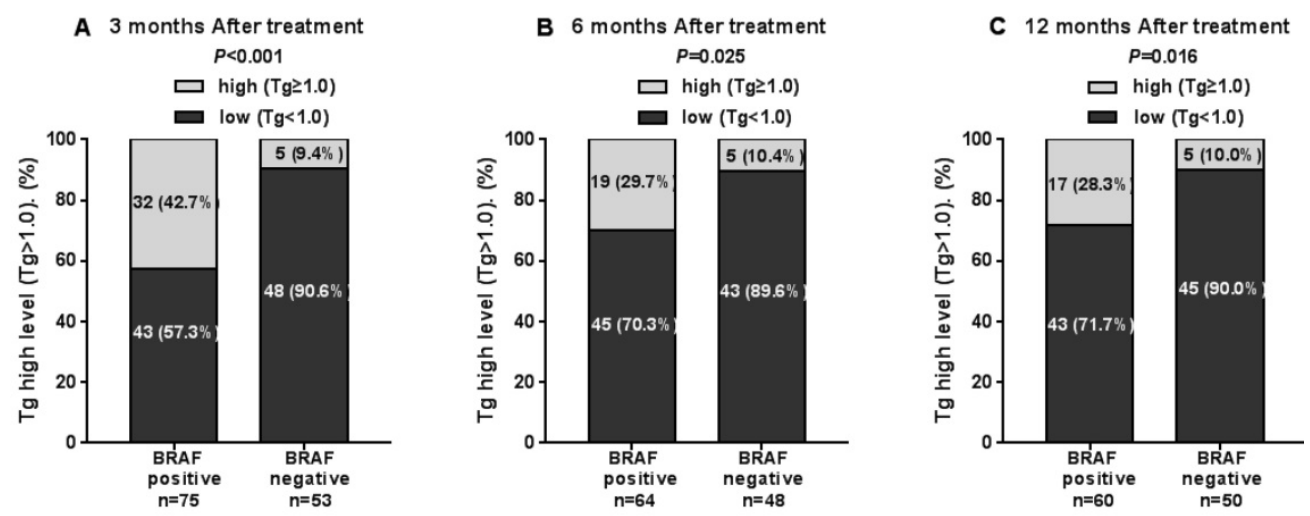

D 18 months After treatment

E 24 months after treatment
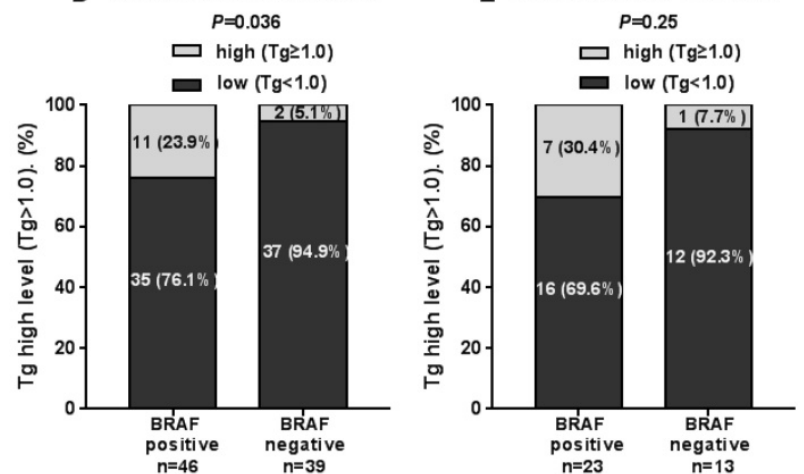

Figure 2. The rate of elevated $\mathrm{Tg}$ levels in the $3 \mathrm{rd}, 6 \mathrm{th}, 12 \mathrm{th}, 18 \mathrm{th}$ and 24 th months after thyroid cancer treatment with iodine-131. 


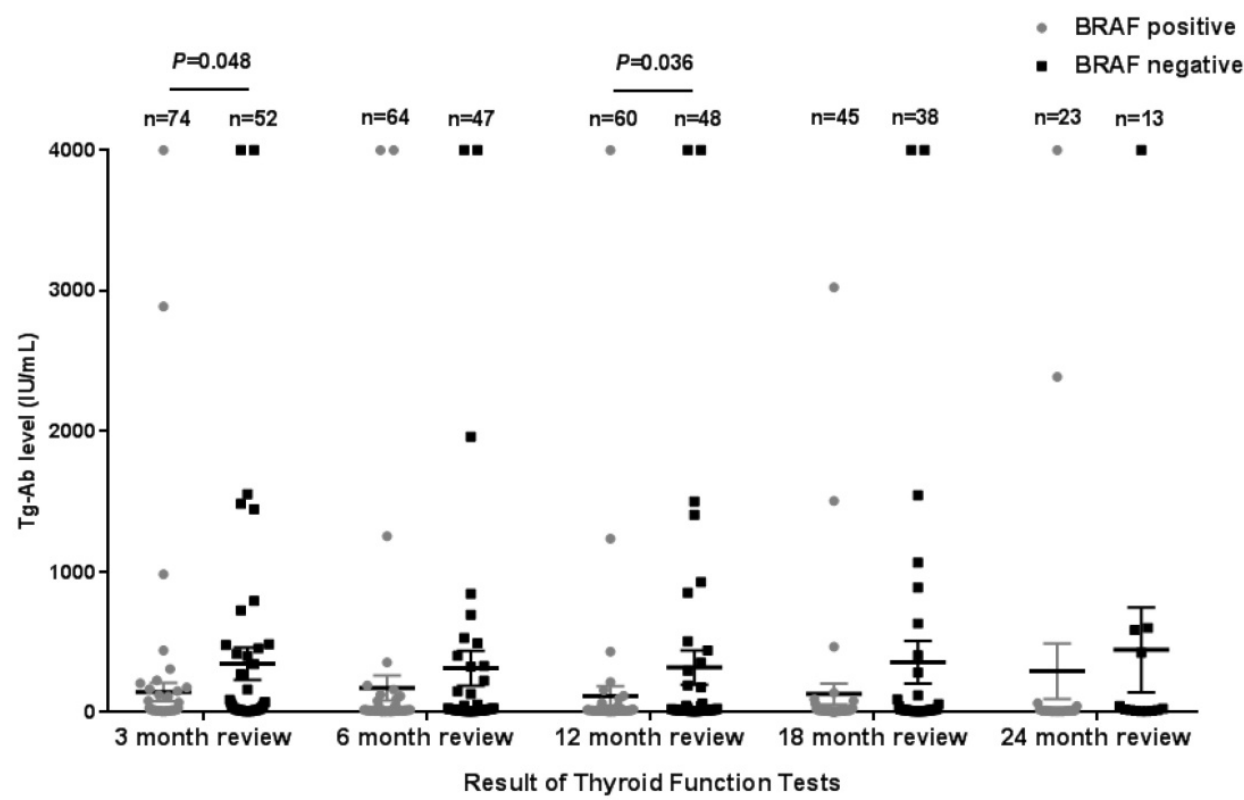

Figure 3. Tg-Ab levels in the 3rd (A), 6th (B), 12th (C)and 18th (D) month after thyroid cancer treatment with iodine-131 in 128 patients from Hunan Cancer Hospital (error bar: mean with SEM).

\section{Discussion}

Thyroid cancer is a common endocrine tumor with an increasing prevalence in China year by year[22-24]. The four primary histological types include papillary carcinoma, medullary carcinoma, undifferentiated carcinoma, and follicular carcinoma [25], among which papillary thyroid cancer (PTC) is the most common, accounting for $85 \%$ to $90 \%$ of the total incidence. The clinical biological characteristics of thyroid cancer with different pathological types are also different. Papillary thyroid carcinoma is one of the less malignant thyroid cancers, which shows low lymph node metastasis rate and distant metastasis rate. Clinically, in addition to the general clinical features, most papillary thyroid carcinoma patients have considerable variable clinical manifestations. This situation may be caused by many factors, including age, gender, living environment, genetic background, and gene mutation.

In recent years, with advances in gene sequencing technology, molecular diagnosis has been widely used for the early diagnosis of cancer, the determination of radiotherapy and chemotherapy, and the postoperative management of patients. In the molecular diagnosis of thyroid cancer, the related molecular markers include TERT, BRAF, PAX8/ PPARY, RAS and RET/PTC[26]. The BRAF V600E gene mutation is one of the most common mutations in PTC $[27,28]$ and is closely related to the occurrence, development, biological behavior and prognosis of PTC $[29,30]$. Studies have shown that an assessment of the BRAF V600E mutational status can be combined with cell morphological analysis to aid in the diagnosis of PTC in cytologically uncertain thyroid nodules[31]. Therefore, we selected 128 patients with PTC who underwent iodine-131 treatment after total thyroidectomy in the Cancer Hospital of Hunan Province from February 2015 to November 2016. We investigated the relationships among the BRAF V600E mutation, clinical pathological features and the efficacy of iodine-131 treatment. Among the 128 patients with PTC included in this study, the BRAF V600E mutation rate was $58.6 \%$. In a previous meta-analysis of BRAF V600E mutation in 25241 cases with PTC, 15290 (60.6\%) were positive for BRAF mutation and 9,951 (39.4\%) were tested negative for BRAF mutation[32]. The inclusion and exclusion criteria for that meta-analysis are basically similar to ours. And the BRAF V600E mutation rate was also similar to our finding.

In this study, we compared the clinical characteristics between patients with the BRAF mutation and patients with wild-type BRAF and found that the BRAF mutation was significantly more frequent in patients of the high-risk age ( $>45$ years old). In the current prognosis evaluation system of thyroid cancer, the patient's age is a recognized risk factor for death; various clinical guidelines and risk assessment models include the patient's age as the primary risk factor in the management and prognostic evaluation of thyroid cancer. The presence of the $B R A F$ mutation is closely related to the patient's age; thus, there may be synergistic effects leading to a poor prognosis[33]. Therefore, in the risk assessment of PTC patients, the BRAF gene mutation and the patient age should be considered as factors associated with a 
poor prognosis. This finding has a certain reference value for the diagnosis and treatment of patients of a high-risk age ( $>45$ years old). However, due to the small sample size and short follow-up time, there was no significant correlation between the BRAF mutation and gender, lymphatic vessel invasion, distant metastasis or clinical stage in this study.

At present, there are few studies on the correlation between BRAF mutational status and iodine-131 treatment, and the results are controversial. According to the guidelines, the current strategies for the treatment of thyroid cancer are primarily surgical treatment, radioactive iodine treatment and TSH inhibition treatment; moleculartargeted therapy has gradually entered the field of vision as an emerging treatment program[34]. The clinical evaluation of the efficacy of iodine-131 treatment typically includes imaging and tests for thyroid function[35]. The key indicators of thyroid function are the levels of thyroglobulin (Tg), thyroid stimulating hormone (TSH), and thyroglobulin antibody (Tg-Ab)[36]. We focused on the relationship between gonadotropin function and BRAF V600E mutational status after iodine-131 treatment.

Patients who were positive for the BRAF V600E mutation had significantly higher $\mathrm{Tg}$ levels compared with patients with wild-type BRAF. $\mathrm{Tg}$ is a glycoprotein that is derived from functional thyroid tissue, accounting for $75 \%$ of all thyroid proteins. $\mathrm{Tg}$ is a macromolecular glycoprotein secreted by thyroid follicular epithelial cells and consists of two identical monomeric peptide chains with a molecular weight of $660 \mathrm{kDa}[37]$. Under normal circumstances, $\mathrm{Tg}$ circulates within thyroid cells, and only a very small portion of the $\mathrm{Tg}$ is secreted into the blood through the thyroid lymphatic vessels. Under normal conditions, the residual tissue size of the thyroid is proportional to the serum levels of Tg. Therefore, after iodine-131 treatment following PTC total resection, the serum levels of $\mathrm{Tg}$ directly reflect the size of the residual thyroid tissue and micrometastases, and an increase in these levels indicate an increase in the residual thyroid tissue and micrometastases[38]. The $\mathrm{Tg}$ level of patients who were positive for the $B R A F$ V600E mutation was significantly increased, suggesting that the therapeutic effect and prognosis of iodine-131 treatment are relatively poor in these patients. Other studies suggest that $\mathrm{Tg}$ level is a key predictor of iodine-131 treatment and PTC prognosis. After iodine-131 treatment following total thyroidectomy, the serum level of thyroglobulin was less than $1 \mu \mathrm{g} / \mathrm{L}$, which is used as the standard for radioactive iodine ablation in patients with low-risk thyroid papillary carcinoma[39]. A Tg level higher than $1 \mu \mathrm{g} / \mathrm{L}$ indicates that the treatment effect of iodine-131 is not good. The rate of elevated Tg levels $(>1.0 \mu \mathrm{g} / \mathrm{L})$ was significantly increased in patients who were positive for the BRAF mutation $(P<0.05)$. This finding indicates that the treatment effect of iodine-131 was worse in these patients than in patients with wild-type BRAF. Recent studies indicate that the BRAF V600E mutation can lead to a downregulation of iodine metabolism because the BRAF V600E mutation leads to the continuous activation of the downstream genes of the MAPK pathway, resulting in the decreased expression and abnormal localization of the sodium iodide symporter (NIS). In the case of wild-type BRAF, NIS is primarily located in the thyroid follicular membrane; however, under conditions of the BRAF V600E mutation, NIS cannot be accurately localized to the follicular membrane and is more dispersed in the cytoplasm[40]. Therefore, patients with the BRAF V600E mutation exhibit poor iodine absorption during iodine-131 treatment, which renders the treatment less effective[41], leading to a poor prognosis. The findings of this study suggest that $B R A F$ mutation can be used as a predictor of the efficacy of iodine-131 treatment.

$\mathrm{Tg}-\mathrm{Ab}$ is a common autoantibody in the serum of patients with autoimmune thyroid disease. This antibody is primarily composed of IgG1, IgG2 and IgG4, and a small portion is IgA and IgM. TG-Ab is generally considered to cause no damage to the thyroid gland. At 3 months and 12 months after iodine-131 treatment, the $\mathrm{Tg}-\mathrm{Ab}$ levels in patients with the BRAF mutation were significantly lower than in patients with wild-type $B R A F$. Regarding the role of $\mathrm{Tg}-\mathrm{Ab}$ in the development of PTC, current research is sparse and contradictory. According to Rakib et al., the trend of postoperative $\mathrm{Tg}-\mathrm{Ab}$ elevation can be used as a rough estimate of prognosis[42, 43]. According to Velsen et al., the continued increase in $\mathrm{Tg}-\mathrm{Ab}$ may suggest tumor recurrence[44]. Smooke-Praw et al. conclude that the level of $\mathrm{Tg}-\mathrm{Ab}$ cannot predict the disease state of PTC[45], whereas $\mathrm{Wu}$ et al. state that the serum levels of $\mathrm{Tg}-\mathrm{Ab}$ can be used as an independent predictor of PTC[46]. The results of the present study suggest that the $B R A F$ mutation may reduce the levels of $\mathrm{Tg}-\mathrm{Ab}$ in the serum, but the specific mechanism warrants further exploration.

Studies have suggested that patients with the BRAF V600E mutation should be overtreated to some extent[47], because BRAF mutations are an independent risk factor for lymph node metastasis and are closely related to tumor size and invasiveness[48-57]. Both the clinical studies and the basic medical studies performed here suggest that the presence of the $B R A F$ mutation may lead to a decrease 
in the efficacy of iodine-131 treatment. Therefore, we suggest that a higher level of radioactive iodine-131 treatment should be considered for patients who are positive for this mutation.

In recent years, individualized treatment has gradually gained attention, and the individualized treatment of cancer has been increasingly promoted[58-63]. As the gene with the highest mutation rate in PTC, the BRAF gene is of great significance for the development of PTC and is widely used in clinical practice. Testing for the BRAF V600E mutation is important, as this mutation is involved in the following: early diagnosis of thyroid cancer with FNC, independent risk factor prediction of PTC high-risk age, independent risk factor prediction of PTC lymph node vascular invasion and lymph node metastasis, prognosis evaluation of PTC, and posttreatment management[64-70]. The results of this study suggest that BRAF mutations should be used as an important indicator when developing individualized treatment options for patients with PTC. According to the results of this study, the BRAF $\mathrm{V} 600 \mathrm{E}$ gene mutation is closely related to serum $\mathrm{Tg}$ elevation after PTC iodine-131 treatment, suggesting that this mutation may be used as a predictor of the efficacy of PTC I-131 treatment.

\section{Abbreviations}

PTC: Papillary Thyroid Cancer; Tg: thyroglobulin; TSH: thyroid stimulating hormone; Tg-Ab: thyroglobulin antibody; Tpo-Ab: thyroid peroxidase antibody; NIS: sodium iodide symporter; ARMS-PCR: amplification-blocking mutation system polymerase chain reaction.

\section{Acknowledgments}

This study was supported by the Hunan provincial natural science fund (2018JJ3313), the Hunan provincial health committee research project (C20180370) and the Sister Institution Network Fund of MD Anderson Cancer Center (2016).

\section{Competing Interests}

The authors have declared that no competing interest exists.

\section{References}

1. Mao Y, Xing M. Recent incidences and differential trends of thyroid cancer in the USA. Endocr Relat Cancer. 2016; 23: 313-22.

2. Doubleday A, Sippel RS. Surgical options for thyroid cancer and post-surgical management. Expert Rev Endocrinol Metab. 2018; 13: 137-48.

3. American Thyroid Association Guidelines Taskforce on Thyroid N, Differentiated Thyroid C, Cooper DS, Doherty GM, Haugen BR, Kloos RT, et al. Revised American Thyroid Association management guidelines for patients with thyroid nodules and differentiated thyroid cancer. Thyroid. 2009; 19: 1167-214.

4. Zhang Y, Xia M, Jin K, Wang S, Wei H, Fan C, et al. Function of the c-Met receptor tyrosine kinase in carcinogenesis and associated therapeutic opportunities. Mol Cancer. 2018; 17: 45.
5. Long GV, Hauschild A, Santinami M, Atkinson V, Mandala M, Chiarion-Sileni $\mathrm{V}$, et al. Adjuvant Dabrafenib plus Trametinib in Stage III BRAF-Mutated Melanoma. N Engl J Med. 2017; 377: 1813-23.

6. Planchard D, Smit EF, Groen HJM, Mazieres J, Besse B, Helland A, et al. Dabrafenib plus trametinib in patients with previously untreated BRAF(V600E)-mutant metastatic non-small-cell lung cancer: an open-label, phase 2 trial. Lancet Oncol. 2017; 18: 1307-16.

7. Corcoran RB, Andre T, Atreya CE, Schellens JHM, Yoshino T, Bendell JC, et al. Combined BRAF, EGFR, and MEK Inhibition in Patients with BRAF(V600E)-Mutant Colorectal Cancer. Cancer Discov. 2018; 8: 428-43.

8. Tu C, Zeng Z, Qi P, Li X, Guo C, Xiong F, et al. Identification of genomic alterations in nasopharyngeal carcinoma and nasopharyngeal carcinoma-derived Epstein-Barr virus by whole-genome sequencing. Carcinogenesis. 2018; 39: 1517-28.

9. Tu C, Zeng Z, Qi P, Li X, Yu Z, Guo C, et al. Genome-Wide Analysis of 18 Epstein-Barr Viruses Isolated from Primary Nasopharyngeal Carcinoma Biopsy Specimens. J Virol. 2017; 91.

10. Xiao L, Wei F, Liang F, Li Q, Deng H, Tan S, et al. TSC22D2 identified as a candidate susceptibility gene of multi-cancer pedigree using genome-wide linkage analysis and whole-exome sequencing. Carcinogenesis. 2019; 40: 819-27.

11. Bai $Y$, Guo T, Huang $X, W u$ Q, Niu D, Ji X, et al. In papillary thyroid carcinoma, expression by immunohistochemistry of BRAF V600E, PD-L1, and PD-1 is closely related. Virchows Arch. 2018; 472: 779-87.

12. Ebina A, Sugitani I, Fujimoto Y, Yamada K. Risk-adapted management of papillary thyroid carcinoma according to our own risk group classification system: is thyroid lobectomy the treatment of choice for low-risk patients? Surgery. 2014; 156: 1579-88; discussion 88-9.

13. Pitoia F, Jerkovich F, Urciuoli C, Schmidt A, Abelleira E, Bueno F, et al. Implementing the Modified 2009 American Thyroid Association Risk Stratification System in Thyroid Cancer Patients with Low and Intermediate Risk of Recurrence. Thyroid. 2015; 25: 1235-42.

14. Yoon JH, Lee HS, Kim EK, Moon HJ, Kwak JY. Malignancy Risk Stratification of Thyroid Nodules: Comparison between the Thyroid Imaging Reporting and Data System and the 2014 American Thyroid Association Management Guidelines. Radiology. 2016; 278: 917-24.

15. Xia M, Zhang $\mathrm{Y}$, Jin $\mathrm{K}$, Lu Z, Zeng Z, Xiong W. Communication between mitochondria and other organelles: a brand-new perspective on mitochondria in cancer. Cell Biosci. 2019; 9: 27.

16. Wang H, Zhou Y, Oyang L, Han Y, Xia L, Lin J, et al. LPLUNC1 stabilises PHB1 by counteracting TRIM21-mediated ubiquitination to inhibit NF-kappaB activity in nasopharyngeal carcinoma. Oncogene. 2019; 38: 5062-75.

17. Fagin JA, Wells SA, Jr. Biologic and Clinical Perspectives on Thyroid Cancer. N Engl J Med. 2016; 375: 1054-67.

18. Riesco-Eizaguirre G, Gutierrez-Martinez P, Garcia-Cabezas MA, Nistal M, Santisteban P. The oncogene BRAF V600E is associated with a high risk of recurrence and less differentiated papillary thyroid carcinoma due to the impairment of $\mathrm{Na}+/ \mathrm{I}$ - targeting to the membrane. Endocr Relat Cancer. 2006; 13: 257-69.

19. Khan N, Oriuchi N, Higuchi T, Zhang H, Endo K. PET in the follow-up of differentiated thyroid cancer. Br J Radiol. 2003; 76: 690-5.

20. Shinohara S, Kikuchi M, Suehiro A, Kishimoto I, Harada H, Hino M, et al. Characteristics and prognosis of patients with thyroglobulin-positive and radioactive iodine whole-body scan-negative differentiated thyroid carcinoma. Jpn J Clin Oncol. 2015; 45: 427-32.

21. Perros P, Boelaert K, Colley S, Evans C, Evans RM, Gerrard Ba G, et al. Guidelines for the management of thyroid cancer. Clin Endocrinol (Oxf). 2014; 81 Suppl 1: 1-122.

22. Wei F, Wu Y, Tang L, Xiong F, Guo C, Li X, et al. Trend analysis of cancer incidence and mortality in China. Sci China Life Sci. 2017; 60: 1271-5.

23. Wu C, Li M, Meng H, Liu Y, Niu W, Zhou Y, et al. Analysis of status and countermeasures of cancer incidence and mortality in China. Sci China Life Sci. 2019; 62: 640-7.

24. Zeng H, Chen W, Zheng R, Zhang S, Ji JS, Zou X, et al. Changing cancer survival in China during 2003-15: a pooled analysis of 17 population-based cancer registries. Lancet Glob Health. 2018; 6: e555-e67.

25. Chmielik E, Rusinek D, Oczko-Wojciechowska M, Jarzab M, Krajewska J, Czarniecka A, et al. Heterogeneity of Thyroid Cancer. Pathobiology. 2018; 85: 117-29.

26. Cabanillas ME, Ferrarotto R, Garden AS, Ahmed S, Busaidy NL, Dadu R, et al. Neoadjuvant BRAF- and Immune-Directed Therapy for Anaplastic Thyroid Carcinoma. Thyroid. 2018; 28: 945-51.

27. Mathur A, Moses W, Rahbari R, Khanafshar E, Duh QY, Clark O, et al. Higher rate of BRAF mutation in papillary thyroid cancer over time: a single-institution study. Cancer. 2011; 117: 4390-5.

28. Chakraborty A, Narkar A, Mukhopadhyaya R, Kane S, D'Cruz A, Rajan MG. BRAF V600E mutation in papillary thyroid carcinoma: significant association with node metastases and extra thyroidal invasion. Endocr Pathol. 2012; 23: 83-93.

29. Pelizzo MR, Dobrinja C, Casal Ide E, Zane M, Lora O, Toniato A, et al. The role of $\mathrm{BRAF}(\mathrm{V} 600 \mathrm{E})$ mutation as poor prognostic factor for the outcome of patients with intrathyroid papillary thyroid carcinoma. Biomed Pharmacother. 2014; 68: 413-7. 
30. Lee MY, Ku BM, Kim HS, Lee JY, Lim SH, Sun JM, et al. Genetic Alterations and Their Clinical Implications in High-Recurrence Risk Papillary Thyroid Cancer. Cancer Res Treat. 2017; 49: 906-14.

31. Beisa A, Kvietkauskas M, Beisa V, Stoskus M, Ostaneviciute E, Jasiunas E, et al. Significance of BRAF V600E Mutation and Cytomorphological Features for the Optimization of Papillary Thyroid Cancer Diagnostics in Cytologically Indeterminate Thyroid Nodules. Exp Clin Endocrinol Diabetes. 2019; 127: 247-54

32. Zhang Q, Liu SZ, Zhang Q, Guan YX, Chen QJ, Zhu QY. Meta-Analyses of Association Between BRAF(V600E) Mutation and Clinicopathological Features of Papillary Thyroid Carcinoma. Cell Physiol Biochem. 2016; 38: 763-76.

33. Shen X, Zhu G, Liu R, Viola D, Elisei R, Puxeddu E, et al. Patient Age-Associated Mortality Risk Is Differentiated by BRAF V600E Status in Papillary Thyroid Cancer. J Clin Oncol. 2018; 36: 438-45.

34. Chau NG, Haddad RI. Vandetanib for the treatment of medullary thyroid cancer. Clin Cancer Res. 2013; 19: 524-9.

35. He Y, Pan MZ, Huang JM, Xie P, Zhang F, Wei LG. Iodine-131: An Effective Method for Treating Lymph Node Metastases of Differentiated Thyroid Cancer. Med Sci Monit. 2016; 22: 4924-8.

36. Faix JD, Miller WG. Progress in standardizing and harmonizing thyroid function tests. Am J Clin Nutr. 2016; 104 Suppl 3: 913S-7S.

37. Netzel BC, Grebe SK, Carranza Leon BG, Castro MR, Clark PM, Hoofnagle AN, et al. Thyroglobulin (Tg) Testing Revisited: Tg Assays, TgAb Assays, and Correlation of Results With Clinical Outcomes. J Clin Endocrinol Metab. 2015; 100: E1074-83.

38. Diaz RE, Veliz J, Wohllk N. [Preablative serum thyroglobulin as predictor of disease-free survival in differentiated thyroid cancer]. Rev Med Chil. 2013; 141: 1506-11

39. Rosario PW, Mineiro Filho AF, Prates BS, Silva LC, Calsolari MR. Postoperative stimulated thyroglobulin of less than $1 \mathrm{ng} / \mathrm{ml}$ as a criterion to spare low-risk patients with papillary thyroid cancer from radioactive iodine ablation. Thyroid. 2012; 22: 1140-3.

40. Tavares C, Coelho MJ, Eloy C, Melo M, da Rocha AG, Pestana A, et al. NIS expression in thyroid tumors, relation with prognosis clinicopathological and molecular features. Endocr Connect. 2018; 7: 78-90.

41. Zhang N, Liang J, Lin YS. Unfavorable efficacy to (131)I ablation in BRAFV600E mutant papillary thyroid carcinoma with positive $\mathrm{TgAb}$. Oncotarget. 2017; 8: 97407-15

42. Rakib SA, Sharif SB, Nahar A, Alam SM. Prediction of Thyroid Malignancies by Thyroid Auto Antibodies. Mymensingh Med J. 2018; 27: 585-95.

43. de Meer SGA, Vorselaars W, Kist JW, Stokkel MPM, de Keizer B, Valk GD, et al. Follow-up of patients with thyroglobulin-antibodies: Rising Tg-Ab trend is a risk factor for recurrence of differentiated thyroid cancer. Endocr Res. 2017; 42: $302-10$

44. van Velsen EFS, Stegenga MT, van Kemenade FJ, Kam BLR, van Ginhoven TM, Visser WE, et al. Comparing the Prognostic Value of the Eighth Edition of the American Joint Committee on Cancer/Tumor Node Metastasis Staging System Between Papillary and Follicular Thyroid Cancer. Thyroid. 2018; 28: 976-81.

45. Smooke-Praw S, Ro K, Levin O, Ituarte PH, Harari A, Yeh MW. Thyroglobulin antibody levels do not predict disease status in papillary thyroid cancer. Clin Endocrinol (Oxf). 2014; 81: 271-5.

46. Wu X, Lun Y, Jiang H, Gang Q, Xin S, Duan Z, et al. Coexistence of thyroglobulin antibodies and thyroid peroxidase antibodies correlates with elevated thyroid-stimulating hormone level and advanced tumor stage of papillary thyroid cancer. Endocrine. 2014; 46: 554-60.

47. Liu L, Chang JW, Jung SN, Park HS, Oh T, Lim YC, et al. Clinical implications of the extent of BRAF(V600E) alleles in patients with papillary thyroid carcinoma. Oral Oncol. 2016; 62: 72-7.

48. Ghaznavi SA, Ganly I, Shaha AR, English C, Wills J, Tuttle RM. Using the American Thyroid Association Risk-Stratification System to Refine and Individualize the American Joint Committee on Cancer Eighth Edition Disease-Specific Survival Estimates in Differentiated Thyroid Cancer. Thyroid. 2018; 28: 1293-300

49. Fan CM, Wang JP, Tang YY, Zhao J, He SY, Xiong F, et al. circMAN1A2 could serve as a novel serum biomarker for malignant tumors. Cancer Sci. 2019; 110: 2180-8.

50. Xiong F, Deng S, Huang HB, Li XY, Zhang WL, Liao QJ, et al. Effects and mechanisms of innate immune molecules on inhibiting nasopharyngeal carcinoma. Chin Med J (Engl). 2019; 132: 749-52.

51. Bo H, Fan L, Gong Z, Liu Z, Shi L, Guo C, et al. Upregulation and hypomethylation of IncRNA AFAP1AS1 predicts a poor prognosis and promotes the migration and invasion of cervical cancer. Oncol Rep. 2019; 41: 2431-9.

52. Bo H, Fan L, Li J, Liu Z, Zhang S, Shi L, et al. High Expression of IncRNA AFAP1-AS1 Promotes the Progression of Colon Cancer and Predicts Poor Prognosis. J Cancer. 2018; 9: 4677-83.

53. Deng X, Xiong F, Li X, Xiang B, Li Z, Wu X, et al. Application of atomic force microscopy in cancer research. J Nanobiotechnology. 2018; 16: 102.

54. Wu Y, Wei F, Tang L, Liao Q, Wang H, Shi L, et al. Herpesvirus acts with the cytoskeleton and promotes cancer progression. J Cancer. 2019;10:2185-93.

55. Fan C, Tu C, Qi P, Guo C, Xiang B, Zhou M, et al. GPC6 Promotes Cell Proliferation, Migration, and Invasion in Nasopharyngeal Carcinoma. J Cancer. 2019; 10:3926-32.
56. Wang W, Zhou R, Wu Y, Liu Y, Su W, Xiong W, et al. PVT1 Promotes Cancer Progression via MicroRNAs. Front Oncol. 2019; 9: 609.

57. Jin K, Wang S, Zhang Y, Xia M, Mo Y, Li X, et al. Long non-coding RNA PVT1 interacts with MYC and its downstream molecules to synergistically promote tumorigenesis. Cell Mol Life Sci. 2019;76:4275-89.

58. Tang L, Wei F, Wu Y, He Y, Shi L, Xiong F, et al. Role of metabolism in cancer cell radioresistance and radiosensitization methods. J Exp Clin Cancer Res. 2018; 37: 87.

59. Wei F, Tang L, He Y, Wu Y, Shi L, Xiong F, et al. BPIFB1 (LPLUNC1) inhibits radioresistance in nasopharyngeal carcinoma by inhibiting VTN expression. Cell Death Dis. 2018; 9: 432

60. He Y, Jing Y, Wei F, Tang Y, Yang L, Luo J, et al. Long non-coding RNA PVT1 predicts poor prognosis and induces radioresistance by regulating DNA repair and cell apoptosis in nasopharyngeal carcinoma. Cell Death Dis. 2018; 9: 235.

61. Mo Y, Wang Y, Zhang L, Yang L, Zhou M, Li X, et al. The role of Wnt signaling pathway in tumor metabolic reprogramming. J Cancer. 2019;10:3789-97.

62. Peng M, Mo Y, Wang $\mathrm{Y}$, Wu P, Zhang $\mathrm{Y}$, Xiong F, et al. Neoantigen vaccine: an emerging tumor immunotherapy. Mol Cancer. 2019;18:128.

63. Mo Y, Wang Y, Xiong F, Ge X, Li Z, Li X, et al. Proteomic Analysis of the Molecular Mechanism of Lovastatin Inhibiting the Growth of Nasopharyngeal Carcinoma Cells. J Cancer. 2019;10: 2342-9.

64. Wei F, Jing YZ, He Y, Tang YY, Yang LT, Wu YF, et al. Cloning and characterization of the putative AFAP1-AS1 promoter region. J Cancer. 2019; 10: $1145-53$

65. Duan S, Guo W, Xu Z, He Y, Liang C, Mo Y, et al. Natural killer group 2D receptor and its ligands in cancer immune escape. Mol Cancer. 2019; 18: 29.

66. Jiang X, Wang J, Deng X, Xiong F, Ge J, Xiang B, et al. Role of the tumor microenvironment in PD-L1/PD-1-mediated tumor immune escape. Mol Cancer. 2019; 18: 10 .

67. Wang YA, Li XL, Mo YZ, Fan CM, Tang L, Xiong F, et al. Effects of tumor metabolic microenvironment on regulatory T cells. Mol Cancer. 2018; 17: 168.

68. Fan C, Tang Y, Wang J, Wang Y, Xiong F, Zhang S, et al. Long non-coding RNA LOC284454 promotes migration and invasion of nasopharyngeal carcinoma via modulating the Rho/Rac signaling pathway. Carcinogenesis. 2019; 40: 380-91.

69. Lian Y, Xiong F, Yang L, Bo H, Gong Z, Wang Y, et al. Long noncoding RNA AFAP1-AS1 acts as a competing endogenous RNA of miR-423-5p to facilitate nasopharyngeal carcinoma metastasis through regulating the Rho/Rac pathway. J Exp Clin Cancer Res. 2018; 37: 253.

70. Fan C, Tang Y, Wang J, Xiong F, Guo C, Wang Y, et al. The emerging role of Epstein-Barr virus encoded microRNAs in nasopharyngeal carcinoma. J Cancer. 2018; 9: 2852-64. 